\title{
Integro-differential equation for brane-world cosmological perturbations
}

\author{
Shinji Mukohyama \\ Department of Physics and Astronomy, University of Victoria \\ Victoria, BC, Canada V8W $3 P 6$
}

\begin{abstract}
Cosmological perturbations in the brane-world cosmology with a positive tension brane in the AdS background bulk geometry is analyzed by using the doubly gauge-invariant formalism. We derive four independent equations for scalar perturbations in the plane symmetric $(K=0)$ background. Three of these equations are differential equations written in terms of gauge invariant variables on the brane only, and another of them is an integro-differential equation whose kernel is constructed formally from the $\mathbf{Z}_{2}$-symmetric retarded Green's function of the bulk gravitational waves. We compare these four equations with the corresponding equations in the standard cosmology. As a by-product, we also obtain a set of equations which may be useful in numerical calculations.
\end{abstract}

\section{INTRODUCTION}

The idea that our four-dimensional world may be a timelike surface, or a world-volume of a 3-brane, in a higher dimensional spacetime has been attracting a great deal of physical interests. As shown by Randall and Sundrum [1], the 4-dimensional Newton's law of gravity can be reproduced on a 4-dimensional timelike hypersurface with positive tension in a 5-dimensional AdS background despite the existence of the infinite fifth dimension.

Moreover, in refs. [2 7] it was shown that the standard cosmology can be realized in the scenario in low energy as far as a spatially homogeneous and isotropic brane is concerned. Hence, it seems interesting to look for observable consequences of the cosmology based on this scenario. For this purpose, cosmic microwave background (CMB) anisotropy seems a powerful tool. In this respect, many authors investigated cosmological perturbations in the braneworld scenario 8 17.

In this paper, we investigate classical evolution of brane-world cosmological perturbations. It was conjectured in ref. [8] that the evolution of cosmological perturbations becomes non-local in the brane-world in the sense that it should be described by some integro-differential equations. The non-locality is due to gravitational waves propagating in the bulk 5-dimensional spacetime. Thus, the main purpose of this paper is to give a formal derivation of an integro-differential equation for brane-world cosmological perturbations. Actually, we shall derive four independent equations for scalar perturbations on the brane, one of which is the integro-differential equation, by using the doubly gauge-invariant formalism developed by the author 8 , 9].

In section III we review the background cosmological solutions in the brane-world scenario and describe the background we use in this paper. In section $\mathrm{III}$, specializing to scalar perturbations around a plane-symmetric $(K=0)$ background, we review the doubly gauge invariant formalism of brane-world cosmological perturbations. Section IV is the main part of this paper. In the section we derive the four equations for perturbatious on the brane including the integro-differential equation. In section $\mathrm{V}$ we compare these four equations in the brane-world cosmology with the corresponding equations in the standard cosmology. Finally, section V1 is devoted to a summary of this paper.

\section{BACKGROUND GEOMETRY}

As mentioned in the introduction, cosmological solutions in the Randall-Sundrum brane world scenario were recently found [4] In these solutions, the standard cosmology is restored in low energy, if a parameter in the solutions is small enough. If the parameter is not small enough, it affects cosmological evolution of our universe as dark radiation [5]. Hence, the parameter should be very small in order that the brane-world scenario should be consistent with nucleosynthesis [⿴囗十 . On the other hand, in ref. [18], it was shown that 5-dimensional geometry of all these cosmological solutions is the Schwarzschild-AdS (S-AdS) spacetime [19] and that the parameter is equivalent to the mass of the black hole. Therefore, the 5-dimensional bulk geometry should be the S-AdS spacetime with a small mass, which is close to the pure AdS spacetime. Moreover, black holes with small mass will evaporate in a short time scale [20]. Thus, it seems a good approximation to consider the pure AdS spacetime as a 5-dimensional bulk geometry for the brane-world cosmology. 
Now let us review the above by using several equations and describe the background geometry which we shall consider throughout this paper. First, let us consider a five-dimensional bulk metric of the form

$$
d s_{5}^{2}=-N^{2}(\tau, w) d \tau^{2}+r^{2}(\tau, w) d \Sigma_{K}^{2}+d w^{2},
$$

where $d \Sigma_{K}^{2}$ is a metric of a unit three-dimensional sphere, plane or hyperboloid for $K=+1,0$ or -1 , respectively. This metric represents a general five-dimensional spacetime with the symmetry of four-dimensional Friedmann universe. Actually, the induced metric on the constant- $w$ hypersurface $\left(w=w_{0}\right)$ is of the four-dimensional Friedmann metric, provided that the new time coordinate $t$ and the scale factor $a(t)$ are introduced by $d t=N\left(\tau, w_{0}\right) d \tau$ and $a(t)=$ $r\left(\tau, w_{0}\right)$. Hence, the metric (1) represents the bulk geometry for the brane-world cosmology in the Gaussian normal coordinate system and $w$ represents the extra dimension if we impose the $Z_{2}$-symmetry, or the invariance under the parity transformation $w-w_{0} \rightarrow w_{0}-w$. It is possible to solve the five-dimensional Einstein equations with the negative cosmological constant by using this form of the metric. The bulk solution and the corresponding cosmological evolution equation induced on the brane were obtained in refs. [4, 4 . 5 .

Instead, we may consider a different coordinate system in which the metric is of the following form 1 .

$$
d s_{5}^{2}=-F(T, r) l^{2} d T^{2}+G(T, r) d r^{2}+r^{2} d \Sigma_{K}^{2} .
$$

In this coordinate system, the five-dimensional Einstein equations with the negative cosmological constant can be solved more easily than in the Gaussian normal coordinate. The solution is the S-AdS spacetime [19]:

$$
F=G^{-1}=K+\frac{r^{2}}{l^{2}}-\frac{\mu}{r^{2}},
$$

where $l$ is the length scale of the five-dimensional negative cosmological constant and $\mu$ is a constant. This statement may be considered as a generalized Birkoff's theorem 2 . Thus, the bulk geometry is static, regardless of the dynamical motion of the brane, insofar as the world-volume of the brane has the symmetry of the three-dimensional sphere, plane or hyperboloid, respectively. The $Z_{2}$-symmetry is incorporated by considering two copies of the same region in the S-AdS spacetime with a timelike boundary and gluing these along the boundary. The corresponding cosmological evolution equation induced on the brane was obtained in refs. [6,7] and is the same as that obtained by using the Gaussian normal coordinate system.

It is, of course, possible to obtain explicit coordinate transformation between the Gaussian normal coordinate system and the spherically, plane or hyperbolically symmetric coordinate system. Actually, in ref. [18] the coordinate transformation was obtained and the global structure of the solutions was investigated by using it.

As pointed out in ref. [5] and explained in the first paragraph of this section, consideration of the dark radiation and the Hawking radiation makes us to think that the pure AdS spacetime, which corresponds to $\mu=0$, should be good enough for the bulk geometry. The generalized Birkoff's theorem guarantees that the AdS bulk spacetime is perfectly consistent with any motion of the brane, provided that the induced geometry on the brane has the symmetry of the three-dimensional sphere, plane or hyperboloid. Hence, throughout this paper, we shall consider the pure AdS spacetime as the bulk geometry. Furthermore, for simplicity we shall consider the spatially flat $(K=0)$ brane only. In this case, it is convenient to introduce a new spatial coordinate $X$ by $X=l / r$ so that the metric (2) with $\mu=K=0$ becomes manifestly conformally flat:

$$
d s_{5}^{2}=g_{M N}^{(0)} d x^{M} d x^{N}=\left(\frac{l}{X}\right)^{2}\left[-d T^{2}+d X^{2}+\sum_{i=1}^{3}\left(d x^{i}\right)^{2}\right],
$$

where $M, N=0,1,2,3,4$.

In this bulk spacetime, let us consider a world volume of a 3-brane or, a timelike hypersurface, $\Sigma^{(0)}$ given by the imbedding relation $x^{M}=Z^{(0) M}(y)$, where $y$ represents $\left\{y^{\mu}\right\}(\mu=0,1,2,3)$ and

\footnotetext{
${ }^{1}$ For latter convenience we included $l^{2}$ in front of $d T^{2}$. So, the time variable $T$ in the present paper corresponds to $T / l$ in ref. [18].

${ }^{2}$ Of course, if we consider matter fields 21,22 or quantum corrections 23, 24 in the bulk then the generalized Birkoff's theorem does not hold. In these more general case, the bulk geometry becomes completely dynamical if the induced geometry on the brane is dynamical (eg. expanding). Thus, inclusion of bulk matter fields or quantum corrections is beyond the scope of this paper.
} 


$$
\begin{aligned}
Z^{(0) T} & =\bar{T}(t), \\
Z^{(0) X} & =\bar{X}(t), \\
Z^{(0) i} & =y^{i} \quad(i=1,2,3) .
\end{aligned}
$$

Hereafter, $t=y^{0}$. The induced metric $q_{\mu \nu}^{(0)}$ on $\Sigma^{(0)}$ defined by

$$
\begin{aligned}
q_{\mu \nu}^{(0)}(y) & =\left.g_{M N}^{(0)} e_{\mu}^{(0) M} e_{\nu}^{(0) N}\right|_{x=Z^{(0)}(y)}, \\
e_{\mu}^{(0) M} & =\frac{\partial Z^{(0) M}}{\partial y^{\mu}}
\end{aligned}
$$

can be calculated to be

$$
q_{\mu \nu}^{(0)} d y^{\mu} d y^{\nu}=-d t^{2}+a^{2}(t) \sum_{i=1}^{3}\left(d y^{i}\right)^{2},
$$

where the time coordinate $t=y^{0}$ has been normalized so that $(l \dot{\bar{T}} / \bar{X})^{2}-(l \dot{\bar{X}} / \bar{X})^{2}=1$ and the function $a(t)$ is defined by $a(t)=l / \bar{X}(t)$. This is actually the flat Friedmann-Robertson-Walker metric. The extrinsic curvature of $\Sigma^{(0)}$ defined by

$$
K_{\mu \nu}^{(0)}(y)=\left.\frac{1}{2} e_{\mu}^{(0) M} e_{\nu}^{(0) N} \mathcal{L}_{n^{(0)}} g_{M N}^{(0)}\right|_{x=Z^{(0)}(y)},
$$

where $n^{(0) M}$ is the unit normal to $\Sigma^{(0)}$, is calculated to be

$$
\begin{aligned}
K_{\mu \nu}^{(0)} d y^{\mu} d y^{\nu} & =\mathcal{K}(t) d t^{2}+a^{2}(t) \overline{\mathcal{K}}(t) \sum_{i=1}^{3}\left(d y^{i}\right)^{2}, \\
\mathcal{K}(t) & =\frac{l^{2} H(t)^{2}+l^{2} \dot{H}(t)+1}{l \sqrt{1+l^{2} H(t)^{2}}} \\
\overline{\mathcal{K}}(t) & =-\frac{\sqrt{1+l^{2} H(t)^{2}}}{l} .
\end{aligned}
$$

Here, $H$ is defined by $H=\dot{a} / a$ and the direction of the unit normal vector $n^{M}$ has been chosen so that $\left.n^{(0) M} \partial_{M} r\right|_{x=Z^{(0)}(y)}<0$.

The $Z_{2}$-symmetry can be incorporated by considering two copies of one of the two regions (i) $X>\bar{X}\left(\bar{T}^{-1}(T)\right)$ or (ii) $X<\bar{X}\left(\bar{T}^{-1}(T)\right)$, and gluing the two copies along $\Sigma^{(0)}$. Hence, Israel's junction condition 25] gives the evolution equation of $a(t)$ as

$$
\pm \frac{\sqrt{1+l^{2} H(t)^{2}}}{l}=\frac{\kappa_{5}^{2}}{6}(\rho(t)+\lambda)
$$

and the conservation equation

$$
\dot{\rho}(t)+3 H(t)(\rho(t)+p(t))=0,
$$

where $\kappa_{5}$ is the 5 -dimensional gravitational constant and we have assumed that the surface energy-momentum tensor, or the Lanczos tensor, $S_{\mu \nu}^{(0)}$ of the brane is of the form

$$
S_{\mu \nu}^{(0)}=-\lambda q_{\mu \nu}+T_{\mu \nu}
$$

where $\lambda$ is the vacuum energy density on the brane, or the tension of the brane, and

$$
T_{\nu}^{\mu}=\left(\begin{array}{cccc}
-\rho(t) & 0 & 0 & 0 \\
0 & p(t) & 0 & 0 \\
0 & 0 & p(t) & 0 \\
0 & 0 & 0 & p(t)
\end{array}\right) .
$$


The sign in (10) is plus or minus for the choice (i) or (ii), respectively. In order for the vacuum $\rho=p=0$ to give the nearly static brane $l^{2} H^{2} \ll 1$, it is necessary and sufficient that $\kappa_{5}^{2} \lambda \simeq \pm 6 / l$, where the plus and the minus signs are for the choice (i) and (ii), respectively. Hence, the tension $\lambda$ of the brane should be positive (or negative) for the choice (i) (or (ii), respectively). With this sign of $\lambda$, the evolution equation (10) can be rewritten as

$$
H^{2}=\frac{8 \pi G_{N}}{3}\left(1+\frac{\rho}{2 \lambda}\right) \rho+\frac{\Lambda_{4}}{3},
$$

where $G_{N}=\kappa_{5}^{4} \lambda / 48 \pi$ and $\Lambda_{4}=\kappa_{5}^{4} \lambda^{2} / 12-3 l^{-2}$. Hence, for the choice (i), we obtain the standard Friedmann equation in low energy (namely when $|\rho| \ll \lambda$ ) and the evolution equation (14) can be considered as a generalized Friedmann equation [2] $G_{N}$ is negative and, thus, the cosmology in low energy becomes very different from the standard cosmology [26] 3 . Therefore, in the following we shall consider the choice (i). Namely, we shall consider two copies of the region $X>\bar{X}\left(\bar{T}^{-1}(T)\right)$ in the pure AdS spacetime and glue them along the trajectory $(T, X)=(\bar{T}(t), \bar{X}(t))$ of the brane with positive $\lambda$.

\section{DOUBLY GAUGE-INVARIANT FORMALISM}

In this section, specializing to scalar perturbations 1 around a plane-symmetric $(K=0)$ background, we review the gauge-invariant formalism of gravitational perturbations in the bulk and the doubly gauge-invariant formulation of the perturbed junction condition developed in refs. [8,9]. In the next section we shall apply the formalism to the brane-world cosmological background described in the previous section.

\section{A. Perturbed junction condition}

First, let us consider a general five-dimensional metric $g_{M N}$, general functions $\left\{Z^{M}(y)\right\}$ determining the imbedding of a general world volume $\Sigma$ of the brane, where $y$ denotes 4 dimensional coordinates $\left\{y^{\mu}\right\}$ on $\Sigma$, and a general surface energy-momentum tensor $S_{\mu \nu}$ on $\Sigma$. We then expand them around a general background specified by $g_{M N}^{(0)}$, $\left\{Z^{(0) M}(y)\right\}$ and $S_{\mu \nu}^{(0)}$. Namely, we consider the metric

$$
d s_{5}^{2}=g_{M N} d x^{M} d x^{N}=\left(g_{M N}^{(0)}+\delta g_{M N}\right) d x^{M} d x^{N},
$$

the imbedding relation

$$
x^{M}=Z^{M}(y)=Z^{(0) M}(y)+\delta Z^{M}(y),
$$

and the surface energy momentum tensor

$$
S_{\mu \nu}=S_{\mu \nu}^{(0)}+\delta S_{\mu \nu}
$$

We can calculate the perturbed induced metric and the perturbed extrinsic curvature of $\Sigma$ up to the linear order and obtain the following expressions.

$$
\begin{aligned}
q_{\mu \nu} & =q_{\mu \nu}^{(0)}+\delta q_{\mu \nu}, \\
K_{\mu \nu} & =K_{\mu \nu}^{(0)}+\delta K_{\mu \nu}
\end{aligned}
$$

where

\footnotetext{
${ }^{3}$ If we consider matter fields or quantum effects in the bulk then this conclusion might be significantly changed since the near brane geometry in the bulk can be very different from AdS spacetime. For analysis of weak gravity in models with bulk matter fields, see ref. 27.

${ }^{4}$ This treatment is, of course, justified since vector and tensor perturbations are decoupled from the scalar perturbations in the linear order. See ref. [5] for the categorization.
} 


$$
\begin{aligned}
\delta q_{\mu \nu}= & e_{\mu}^{(0) M} e_{\nu}^{(0) N}\left(\delta g_{M N}+\mathcal{L}_{\delta Z} g_{M N}^{(0)}\right), \\
\delta K_{\mu \nu}= & \frac{1}{2} n^{(0) M} n^{(0) N}\left(\delta g_{M N}+2 \delta Z_{M ; N}\right) K_{\mu \nu}^{(0)} \\
& -\frac{1}{2} n^{(0) L} e_{\mu}^{(0) M} e_{\nu}^{(0) N}\left[2 \delta \Gamma_{L M N}+\delta Z_{L ; M N}+\delta Z_{L ; N M}+\left(R_{L^{\prime} M L N}^{(0)}+R_{L^{\prime} N L M}^{(0)}\right) \delta Z^{L^{\prime}}\right] .
\end{aligned}
$$

Here, $\mathcal{L}$ denotes the Lie derivative defined in the five-dimensional spacetime, the semicolon denotes the covariant derivative compatible with the background metric $g_{M N}^{(0)}, R_{L^{\prime} M L N}^{(0)}$ is the Riemann tensor of $g_{M N}^{(0)}$ and $\delta \Gamma_{L M N}=$ $(1 / 2)\left(\delta g_{L M ; N}+\delta g_{L N ; M}-\delta g_{M N ; L}\right)$. The perturbations $\delta q_{\mu \nu}$ and $\delta K_{\mu \nu}$ may be evaluated on $\Sigma^{(0)}$.

For these perturbations we have the following two kinds of gauge transformations [9]. We have the 5-gauge transformation (infinitesimal coordinate transformation in the 5-dimensional bulk spacetime), $x^{M} \rightarrow x^{\prime M}=x^{M}+\bar{\xi}^{M}(x)$, under which $\delta g_{M N}$ and $\delta Z^{M}$ transform as

$$
\begin{aligned}
\delta g_{M N} & \rightarrow \delta g_{M N}-\bar{\xi}_{M ; N}-\bar{\xi}_{N ; M}, \\
\delta Z^{M} & \rightarrow \delta Z^{M}+\bar{\xi}^{M},
\end{aligned}
$$

but $\delta q_{\mu \nu}, \delta K_{\mu \nu}$ and $\delta S_{\mu \nu}$ are invariant. We also have the 4-gauge transformation (infinitesimal reparameterization of the 4-dimensional hypersurface $\Sigma), y^{\mu} \rightarrow y^{\prime \mu}=y^{\mu}+\bar{\zeta}^{\mu}(y)$, under which

$$
\begin{aligned}
\delta q_{\mu \nu} & \rightarrow \delta q_{\mu \nu}-\overline{\mathcal{L}}_{\bar{\zeta}} q_{\mu \nu}^{(0)}, \\
\delta K_{\mu \nu} & \rightarrow \delta K_{\mu \nu}-\overline{\mathcal{L}}_{\bar{\zeta}} K_{\mu \nu}^{(0)}, \\
\delta S_{\mu \nu} & \rightarrow \delta S_{\mu \nu}-\overline{\mathcal{L}}_{\bar{\zeta}} S_{\mu \nu}^{(0)}
\end{aligned}
$$

Here, $\overline{\mathcal{L}}$ denotes the Lie derivative defined in the 4-dimensional manifold $\Sigma^{(0)}$. Note that the 4-gauge transformation is not a part of the 5-gauge transformation. Actually, $\delta q_{\mu \nu}$ and $\delta K_{\mu \nu}$ are invariant under the 5-gauge transformation but are not invariant under the 4-gauge transformation. These two gauge transformation were disentangled from each other in ref. [9].

Next, as the background let us consider the plane-symmetric metric

$$
g_{M N}^{(0)} d x^{M} d x^{N}=\gamma_{a b} d x^{a} d x^{b}+r^{2} \sum_{i=1}^{3}\left(d x^{i}\right)^{2},
$$

and such imbedding functions $Z^{(0) M}(y)$ that $Z^{(0) a}$ depends only on $y^{0}$ and that $Z^{(0) i}=y^{i}$, where the two-dimensional metric $\gamma_{a b}$ and the function $r^{2}$ are assumed to depend only on the two dimensional coordinates $\left\{x^{a}\right\}$. Let us call the two-dimensional spacetime spanned by $\left\{x^{a}\right\}$ an orbit space since the unperturbed motion of the brane can be described as an orbit in this two-dimensional spacetime. As in the previous section, we assume that the hypersurface $\Sigma^{(0)}$ is timelike and $y^{0}$ is normalized so that it represents the proper time $t$ along the trajectory of the brane. The background metric (田) and imbedding functions (5) described in the previous section is an example. In the plane symmetric background we can decompose perturbations as follows

$$
\begin{aligned}
\delta g_{M N} d x^{M} d x^{N}= & \int d^{3} \mathbf{k}\left[h_{a b} Y d x^{a} d x^{b}+2 h_{(L) a} V_{(L) i} d x^{a} d x^{i}\right. \\
& \left.+\left(h_{(L L)} T_{(L L) i j}+h_{(Y)} T_{(Y) i j}\right) d x^{i} d x^{j}\right], \\
\delta Z_{M} d x^{M}= & \int d^{3} \mathbf{k}\left[z_{a} Y d x^{a}+z_{(L)} V_{(L) i} d x^{i}\right], \\
\delta S_{\mu \nu} d y^{\mu} d y^{\nu}= & \int d^{3} \mathbf{k}\left[t_{00} Y d y^{0} d y^{0}+2 t_{(L) 0} V_{(L) i} d y^{0} d y^{i}\right. \\
& \left.+\left(t_{(L L)} T_{(L L) i j}+t_{(Y)} T_{(Y) i j}\right) d y^{i} d y^{j}\right],
\end{aligned}
$$

where $Y=\exp (-i \mathbf{k} \cdot \mathbf{x}), V_{(L) i}=\partial_{i} Y, T_{(L L) i j}=2 \partial_{i} \partial_{j} Y+\left(2 \mathbf{k}^{2} / 3\right) \delta_{i j} Y$ and $T_{(Y) i j}=\delta_{i j} Y$, and all coefficients are supposed to depend only on the 2-dimensional coordinates $\left\{x^{a}\right\}$ of the orbit space. Here, $\mathbf{x}$ denotes coordinates $\left\{x^{i}\right\}$ of the three-dimensional plane $(i=1,2,3)$, and $\mathbf{k}$ represents the momentum $\left\{k_{i}\right\}$ along the plane. Hereafter, we omit $\mathbf{k}$ in most cases. It is easy to see how the coefficients $\left\{h^{\prime} s, z^{\prime} s\right\}$ transform under the 5 -gauge transformation by using (20) and to construct 5-gauge-invariant variables, those linear combinations which are invariant under the 5-gauge 
transformation. Therefore, we obtain 5-gauge-invariant variables corresponding to perturbations of physical position of the hypersurface $\Sigma$

$$
\phi_{a}=z_{a}+X_{a}
$$

as well as 5-gauge-invariant variables representing gravitational perturbations in the bulk

$$
\begin{aligned}
F_{a b} & =h_{a b}-\nabla_{a} X_{b}-\nabla_{b} X_{a}, \\
F & =h_{(Y)}-X^{a} \partial_{b} r^{2}+\frac{2 k^{2}}{n} h_{(L L)},
\end{aligned}
$$

where $X_{a}=h_{(L) a}-r^{2} \partial_{a}\left(r^{-2} h_{(L L)}\right)$.

It is also easy to obtain the corresponding expansion of $\delta q_{\mu \nu}$ and $\delta K_{\mu \nu}$ by using the expression (19). After analyzing 4-gauge transformations (21) of coefficients of the expansion, we can construct 4-gauge-invariant variables, those linear combinations that are invariant under the 4-gauge transformation, and express them in terms of 5-gauge-invariant variables as follows. For perturbations of the induced metric, we have 5

$$
\begin{aligned}
f_{00} & =e^{a} e^{b} F_{a b}+2 \mathcal{K} \phi, \\
f & =F+\phi n^{a} \partial_{a} r^{2},
\end{aligned}
$$

where $e^{a}, n^{a}$ and $\phi$ are abbreviations for $e_{0}^{(0) a} n^{(0) a}$ and $n^{a} \phi_{a}$, respectively, and $\mathcal{K}$ denotes the 00-component $K_{00}^{(0)}$ of the unperturbed extrinsic curvature as in (9). For perturbations of the extrinsic curvature, we have

$$
\begin{aligned}
k_{00} & =-\frac{1}{2} n^{a} e^{b} e^{c}\left(2 \nabla_{c} F_{a b}-\nabla_{a} F_{b c}\right)+\frac{1}{2}\left(n^{a} n^{b}+2 e^{a} e^{b}\right) F_{a b} \mathcal{K}-\ddot{\phi}+\frac{1}{2}\left(R^{(\gamma)}+2 \mathcal{K}^{2}\right) \phi, \\
k_{(L) 0} & =-\frac{1}{2} n^{a} e^{b} F_{a b}-r\left(r^{-1} \phi\right)^{\prime}, \\
k_{(L L)} & =-\frac{1}{2} \phi, \\
k_{(Y)} & =\frac{1}{4} F_{a b} n^{a}\left(2 e^{b} e^{c}-n^{b} n^{c}\right) \partial_{c} r^{2}+\frac{1}{2} r^{2} n^{a} \partial_{a}\left(r^{-2} F\right)+\frac{1}{2} \dot{\phi} e^{c} \partial_{c} r^{2}+\phi\left(r^{2} n^{b} n^{c} \nabla_{b} \nabla_{c} \ln r+\frac{\mathbf{k}^{2}}{3}\right),
\end{aligned}
$$

where $\nabla_{a}$ is the two-dimensional covariant derivative compatible with the metric $\gamma_{a b}$ of the orbit space and $R^{(\gamma)}$ is the Ricci scalar of $\gamma_{a b}$ and dots denote derivative with respect to the proper time $t$. Note that the right hand sides of (26) and (27) are evaluated on the unperturbed hypersurface $\Sigma^{(0)}$ and that $\phi$ can be considered as a function of the proper time $t$.

Now, for completeness, we show the relation among the 4-gauge-invariant variables and $\left(\delta q_{\mu \nu}, \delta K_{\mu \nu}\right)$ in (18).

$$
\begin{aligned}
f_{00} & =\sigma_{00}-2 \dot{\chi}, \\
f & =\sigma_{(Y)}+2 \chi r \dot{r}+\frac{2 k^{2}}{3} \sigma_{(L L)}, \\
k_{00} & =\tilde{\kappa}_{00}+\chi \dot{\mathcal{K}}, \\
k_{(L) 0} & =\tilde{\kappa}_{(L) 0}+\frac{1}{2}(\overline{\mathcal{K}}+\mathcal{K})\left[\chi-r^{2}\left(r^{-2} \sigma_{L L}\right)^{\cdot}\right], \\
k_{(L L)} & =\tilde{\kappa}_{(L L)} \\
k_{(Y)} & =\tilde{\kappa}_{(Y)}+r^{2} \chi \dot{\mathcal{K}},
\end{aligned}
$$

where $\chi=\sigma_{(L) 0}-r^{2}\left(r^{-2} \sigma_{(L L)}\right)^{\cdot}$ and

$$
\begin{aligned}
& \delta q_{\mu \nu} d y^{\mu} d y^{\nu}= \sum_{k}\left[\sigma_{00} Y d y^{0} d y^{0}+2 \sigma_{(L) 0} V_{(L) i} d y^{0} d y^{i}\right. \\
&\left.+\left(\sigma_{(L L)} T_{(L L) i j}+\sigma_{(Y)} T_{(Y) i j}\right) d y^{i} d y^{j}\right] \\
& {\left[\delta K_{\mu \nu}-\frac{1}{2}\left(K_{\mu}^{(0) \rho} \delta q_{\rho \nu}+K_{\nu}^{(0) \rho} \delta q_{\rho \mu}\right)\right] d y^{\mu} d y^{\nu}=} \sum_{k}\left[\tilde{\kappa}_{00} Y d y^{0} d y^{0}+2 \tilde{\kappa}_{(L) 0} V_{(L) i} d y^{0} d y^{i}\right. \\
&\left.+\left(\tilde{\kappa}_{(L L)} T_{(L L) i j}+\tilde{\kappa}_{(Y)} T_{(Y) i j}\right) d y^{i} d y^{j}\right] . \\
&
\end{aligned}
$$

${ }^{5}$ In ref. [9], there is a typo in the sign of the second term of $f_{00}$. 
Finally, the doubly gauge-invariant junction condition with the $Z_{2}$-symmetry becomes

$$
\begin{aligned}
2 k_{00} & =-\kappa^{2}\left(\frac{2}{3} \tau_{00}+a^{-2} \tau_{(Y)}\right), \\
2 k_{(Y)} & =-\frac{1}{3} \kappa^{2} a^{2} \tau_{00}, \\
2 k_{(L) 0} & =-\kappa^{2} \tau_{(L) 0}, \\
2 k_{(L L)} & =-\kappa^{2} \tau_{(L L)},
\end{aligned}
$$

where $\tau_{00}, \tau_{(Y)}, \tau_{(L) 0}$ and $\tau_{(L L)}$ are 4-gauge-invariant variables constructed in ref. [8 from perturbations $\left\{t^{\prime} s\right\}$ of the surface energy momentum tensor on the brane (see the last equation in (23)). The perturbed junction condition (30) was first derived in ref. [10] and rederived in ref. [9].

The relation between the above 4-gauge-invariant variables and gauge-invariant variables which are commonly used in the standard cosmology 2830 is as follows.

$$
\begin{aligned}
f_{00} & =-2 \Psi_{(K S)}, \\
f & =2 a^{2} \Phi_{(K S)}, \\
\tau_{00} & =\rho \Delta_{(K S)}+\dot{\rho} \frac{a V_{(K S)}}{|\mathbf{k}|}, \\
\tau_{(Y)} & =a^{2}\left[p \Gamma_{(K S)}+c_{s}^{2}\left(\rho \Delta_{(K S)}+\dot{\rho} \frac{a V_{(K S)}}{|\mathbf{k}|}\right)\right], \\
\tau_{(L) 0} & =(\rho+p) \frac{a V_{(K S)}}{|\mathbf{k}|}, \\
\tau_{(L L)} & =a^{2} p \frac{\Pi_{(K S)}}{2 \mathbf{k}^{2}},
\end{aligned}
$$

where variables with the subscript $(K S)$ are those defined in ref. [29] and $c_{s}$ is the sound velocity defined by $c_{s}^{2}=\dot{p} / \dot{\rho}$.

\section{B. Master equation in the bulk}

As shown first in ref. [8] and confirmed in ref. [10] $]^{6}$, all components of perturbed Einstein's equation in the bulk around the pure AdS background can be reduced to a single equation called the master equation. For $K=0$ and $\mathbf{k} \neq 0$, the master equation is of the form

$$
\mathbf{L}_{\mathbf{k}} \Phi=0,
$$

where $\Phi$ is the master variable depending only on the coordinates $\left\{x^{a}\right\}$ in the two-dimensional orbit space and the linear differential operator $\mathbf{L}_{\mathbf{k}}$ is defined by

$$
\mathbf{L}_{\mathbf{k}} \Psi \equiv r^{2} \nabla^{a}\left[r^{-1} \nabla_{a}\left(r^{-1} \Psi\right)\right]-\mathbf{k}^{2} r^{-2} \Psi .
$$

For example, in the conformally flat coordinate system considered in section I the master equation becomes

$$
X^{-2} \partial_{X}\left[X \partial_{X}(X \Phi)\right]-\partial_{T}^{2} \Phi-\mathbf{k}^{2} \Phi=0
$$

and is easily solved. The general solution is

$$
\Phi=\frac{1}{X}\left\{e^{-i|\mathbf{k}| T}(C \ln X+D)+\int_{0}^{\infty} d \mu e^{-i \sqrt{\mathbf{k}^{2}+\mu^{2}} T}\left[A(\mu) J_{0}(\mu X)+B(\mu) Y_{0}(\mu X)\right]+(c . c)\right\},
$$

where (c.c) represents the complex conjugate.

\footnotetext{
${ }^{6}$ In the latter paper, they extended the master equation of vector and tensor perturbations in the bulk to more general background without maximal symmetry.
} 
Actually, 5-gauge-invariant variables for scalar perturbations are written in terms of the master variable as

$$
\begin{aligned}
F_{a b} & =\frac{1}{r}\left(\nabla_{a} \nabla_{b} \Phi-\frac{2}{3} \nabla^{2} \Phi \gamma_{a b}+\frac{1}{3 l^{2}} \Phi \gamma_{a b}\right), \\
F & =\frac{r}{3}\left(\nabla^{2} \Phi-\frac{2}{l^{2}} \Phi\right) .
\end{aligned}
$$

For $\mathbf{k}=0$, the corresponding perturbation has the plane symmetry and, thus, the generalized Birkoff's theorem guarantees that the perturbed bulk geometry is a S-AdS spacetime. Hence, the perturbation corresponding to $\mathbf{k}=0$ is actually perturbation of the parameter $\mu$ in (3) around $\mu=0$ and can be understood as dark radiation [5]. Since the effect of non-zero $\mu$ has already been analyzed non-perturbatively in refs. [2 [7], in this paper we shall concentrate on perturbations with non-zero $\mathbf{k}$. This treatment is, of course, justified since perturbations with different $\mathbf{k}$ are decoupled from each other in the linear order.

\section{EQUATIONS ON THE BRANE}

As we have seen in the previous section, all 5-gauge-invariant variables and 4-gauge-invariant variables are written in terms of the master variable $\Phi$. After some cumbersome calculations using the master equation (32), we find the following set of three equations among the 4-gauge-invariant geometrical variables.

$$
\begin{array}{r}
\dot{k}_{(L) 0}+3 H k_{(L) 0}-k_{00}+2 a^{-2} k_{(Y)}+\frac{l \dot{H}}{2 \sqrt{1+l^{2} H^{2}}} f_{00}+\frac{4}{3} \mathbf{k}^{2} a^{-2} k_{(L L)}=0, \\
\dot{k}_{(Y)}-H k_{(Y)}+\frac{l \dot{H}}{2 \sqrt{1+l^{2} H^{2}}}(\dot{f}-2 H f)+\frac{1}{3} \mathbf{k}^{2} k_{(L) 0}+a^{2} H k_{00}=0,
\end{array}
$$

and

$$
\begin{aligned}
\ddot{f}+a^{2} H \dot{f}_{00} & +2\left(2 H^{2}+\dot{H}\right)\left(a^{2} f_{00}-f\right)+\frac{\mathbf{k}^{2}}{3 a^{2}}\left(2 f-a^{2} f_{00}\right) \\
- & \frac{2 a^{2} \sqrt{1+l^{2} H^{2}}}{l} k_{00}+\frac{2\left(3+3 l^{2} H^{2}+l^{2} \dot{H}\right)}{l \sqrt{1+l^{2} H^{2}}} k_{(Y)}=0 .
\end{aligned}
$$

Note that the equations (37) represent nothing but the gauge-invariant expression of perturbation of the equation

$$
\nabla_{\nu}^{(4)}\left(K_{\mu}^{\nu}-K \delta_{\mu}^{\nu}\right)=R_{M N} e_{\mu}^{M} n^{N}=0
$$

where $\nabla_{\mu}^{(4)}$ is the four-dimensional covariant derivative compatible with the induced metric $q_{\mu \nu}$. On the other hand, (38) is the gauge-invariant expression of perturbation of the equation 7

$$
K^{2}-K_{\nu}^{\mu} K_{\mu}^{\nu}-R^{(4)}=2 G_{M N} n^{M} n^{N}=12 l^{-2},
$$

and, as we shall see, can be interpreted as an evolution equation of $f$ after use of the junction condition. Here, $R^{(4)}$ is the Ricci scalar of the four-dimensional induced metric $q_{\mu \nu}$. Furthermore, we can express the master variable and its derivative at any point on the brane in terms of the 4-gauge-invariant geometrical variables. For example,

$$
\begin{aligned}
\dot{f}-2 H f+\frac{\mathbf{k}^{2}}{3 a^{2} H} f+a^{2} H f_{00}+\frac{2 \sqrt{1+l^{2} H^{2}}}{l H} k_{(Y)} & =\left.\frac{\mathbf{k}^{4}}{9 a^{3} H} \Phi\right|_{x^{a}=Z^{(0) a}(t)}, \\
k_{(Y)}+a^{2} H k_{(L) 0}+\frac{2}{3} \mathbf{k}^{2} k_{(L L)} & =\left.\frac{\mathbf{k}^{2}}{6 a} r \partial_{\perp}\left(r^{-1} \Phi\right)\right|_{x^{a}=Z^{(0) a}(t)},
\end{aligned}
$$

where $\partial_{\perp}=n^{a} \partial_{a}$. If one like, one can check these equations (37), (38) and (41) by using the general solution (35) and a computer algebra package like GRTensor II [31].

\footnotetext{
${ }^{7}$ The author thanks W. Israel for pointing this out.
} 
By substituting the doubly gauge-invariant junction condition (30), we obtain the perturbed conservation equation

$$
\begin{array}{r}
\dot{\tau}_{(L) 0}+3 H \tau_{(L) 0}-\frac{1}{a^{2}} \tau_{(Y)}+\frac{4}{3} \frac{\mathbf{k}^{2}}{a^{2}} \tau_{(L L)}-\frac{l \dot{H}}{\kappa_{5}^{2} \sqrt{1+l^{2} H^{2}}} f_{00}=0, \\
\dot{\tau}_{00}+3 H \tau_{00}+\frac{3 H}{a^{2}} \tau_{(Y)}+\frac{\mathbf{k}^{2}}{a^{2}} \tau_{(L) 0}-\frac{3 l \dot{H}}{\kappa_{5}^{2} a^{2} \sqrt{1+l^{2} H^{2}}}(\dot{f}-2 H f)=0,
\end{array}
$$

the evolution equation of $f$

$$
\begin{aligned}
\ddot{f}+a^{2} H \dot{f}_{00} & +2\left(2 H^{2}+\dot{H}\right)\left(a^{2} f_{00}-f\right)+\frac{\mathbf{k}^{2}}{3 a^{2}}\left(2 f-a^{2} f_{00}\right) \\
& +\frac{\kappa_{5}^{2} \sqrt{1+l^{2} H^{2}}}{l} \tau_{(Y)}-\frac{\kappa_{5}^{2} a^{2}\left(1+l^{2} H^{2}+l^{2} \dot{H}\right)}{3 l \sqrt{1+l^{2} H^{2}}} \tau_{00}=0
\end{aligned}
$$

and the expressions of $\Phi$ and $r \partial_{\perp}\left(r^{-1} \Phi\right)$ on the brane

$$
\begin{aligned}
\dot{f}-2 H f+\frac{\mathbf{k}^{2}}{3 a^{2} H} f+a^{2} H f_{00}-\frac{1}{3} \kappa_{5}^{2} a^{2} \frac{\sqrt{1+l^{2} H^{2}}}{l H} \tau_{00} & =\left.\frac{\mathbf{k}^{4}}{9 a^{3} H} \Phi\right|_{x^{a}=Z^{(0) a}(t)}, \\
\tau_{00}+3 H \tau_{(L) 0}+\frac{2 \mathbf{k}^{2}}{a^{2}} \tau_{(L L)} & =-\left.\frac{\mathbf{k}^{2}}{\kappa_{5}^{2} a^{3}} r \partial_{\perp}\left(r^{-1} \Phi\right)\right|_{x^{a}=Z^{(0) a}(t)} .
\end{aligned}
$$

Now we shall use the equation (45) to give a boundary condition to $\Phi$ and use another equation (44) to give a feedback of $\Phi$ to perturbations on the brane. First, let us introduce the $Z_{2}$-symmetric retarded Green's function $G_{\mathbf{R}}\left(x^{a}, x^{\prime a}\right)$ satisfying

$$
\mathbf{L}_{\mathbf{k}} G_{\mathbf{R}}\left(x^{a},{x^{\prime}}^{a}\right)=\frac{\delta^{2}\left(x^{a}-{x^{\prime}}^{a}\right)}{\sqrt{-\gamma}}
$$

in the $\mathbf{Z}_{2}$-symmetrized orbit space. Here, the $\mathbf{Z}_{2}$-symmetrized orbit space is obtained from two copies of the region (i) of the AdS spacetime glued together along the unperturbed world volume $\Sigma^{(0)}$ of the brane (see section 国). Hence, $\Phi$ is expressed as

$$
\Phi\left(x^{a}\right)=\Phi_{i n}\left(x^{a}\right)-2 \int d t^{\prime} G_{\mathbf{R}}\left(x^{a}, Z^{(0) a}\left(t^{\prime}\right)\right)\left[\frac{\kappa_{5}^{2} a\left(t^{\prime}\right)^{3}}{\mathbf{k}^{2}} S\left(t^{\prime}\right)+\left.r \partial_{\perp}\left(r^{-1} \Phi_{i n}\right)\right|_{x^{a}=Z^{(0) a}\left(t^{\prime}\right)}\right],
$$

where $\Phi_{i n}\left(x^{a}\right)$ is an arbitrary solution of the master equation (32) representing initial gravitational waves in the bulk and $S(t)$ is the left hand side of (45). Hence, by substituting this into (44) we obtain

$$
\begin{aligned}
& R(t)-\frac{\mathbf{k}^{4}}{9 a(t)^{3} H(t)} \Phi_{i n}\left(Z^{(0) a}(t)\right) \\
& +\frac{2 \mathbf{k}^{2}}{9 a(t)^{3} H(t)} \int d t^{\prime} \bar{G}_{\mathbf{R}}\left(t, t^{\prime}\right)\left[\kappa_{5}^{2} a\left(t^{\prime}\right)^{3} S\left(t^{\prime}\right)+\left.\mathbf{k}^{2} r \partial_{\perp}\left(r^{-1} \Phi_{i n}\right)\right|_{x^{a}=Z^{(0) a}\left(t^{\prime}\right)}\right]=0,
\end{aligned}
$$

where $\bar{G}_{\mathbf{R}}\left(t, t^{\prime}\right)=G_{\mathbf{R}}\left(Z^{(0) a}(t), Z^{(0) a}\left(t^{\prime}\right)\right)$ and $R(t)$ is the left hand side of (44). This is the integro-differential equation which we have been seeking since conjectured in ref. [8]. If there is no initial gravitational waves in the bulk, then the integro-differential equation is reduced to

$$
R(t)+\frac{2 \kappa_{5}^{2} \mathbf{k}^{2}}{9 a(t)^{3} H(t)} \int d t^{\prime} \bar{G}_{\mathbf{R}}\left(t, t^{\prime}\right) a\left(t^{\prime}\right)^{3} S\left(t^{\prime}\right)=0 .
$$

Finally, we have found the set of four coupled equations (42), 443) and (48). These equations are written in terms of physical quantities on the brane and can be used to determine the evolution of cosmological perturbations on the brane. Note that the number of equations, four, is the same as the number of independent components of perturbed Einstein's equation for scalar perturbations in the standard cosmology as we shall see in the next section.

Although we have succeeded in deriving the integro-differential equation formally, we need an explicit expression of the $\mathbf{Z}_{2}$-symmetric retarded Green's function in order to obtain the kernel. Since evaluation of the Green's function for a general motion of the brane seems difficult, it might be more realistic to adopt numerical methods to integrate the master equation (32) in the bulk and the three differential equations on the brane, (42) and (43), at the same time. In this case, we can use the equations (45) and (44) to give a boundary condition to $\Phi$ and to give a feedback of $\Phi$ to perturbations on the brane. 


\section{COMPARISON WITH THE STANDARD COSMOLOGY}

In this section we compare the four independent equations derived in the previous section with the corresponding equations in the standard cosmology.

\section{A. Brane-world cosmology}

By using the background junction condition (10) with the plus sign and the conservation equation (11), we can rewrite the four equations obtained in the previous section as follows. The conservation equation is

$$
\begin{array}{r}
\dot{\tau}_{(L) 0}+3 H \tau_{(L) 0}-\frac{1}{a^{2}} \tau_{(Y)}+\frac{4}{3} \frac{\mathbf{k}^{2}}{a^{2}} \tau_{(L L)}+\frac{1}{2}(\rho+p) f_{00}=0, \\
\dot{\tau}_{00}+3 H \tau_{00}+\frac{3 H}{a^{2}} \tau_{(Y)}+\frac{\mathbf{k}^{2}}{a^{2}} \tau_{(L) 0}+\frac{3}{2 a^{2}}(\rho+p)(\dot{f}-2 H f)=0,
\end{array}
$$

the evolution equation of $f$ is

$$
\begin{aligned}
\ddot{f}+a^{2} H \dot{f}_{00} & +2\left(2 H^{2}+\dot{H}\right)\left(a^{2} f_{00}-f\right)+\frac{\mathbf{k}^{2}}{3 a^{2}}\left(2 f-a^{2} f_{00}\right) \\
& +8 \pi G_{N}\left(1+\frac{\rho}{\lambda}\right) \tau_{(Y)}-\frac{8 \pi G_{N}}{3}\left(1-\frac{2 \rho+3 p}{\lambda}\right) a^{2} \tau_{00}=0
\end{aligned}
$$

and the integro-differential equation without initial gravitational waves in the bulk, $\Phi_{\text {in }}=0$, is

$$
R(t)+\frac{2 \kappa_{5}^{2} \mathbf{k}^{2}}{9 a(t)^{3} H(t)} \int d t^{\prime} \bar{G}_{\mathbf{R}}\left(t, t^{\prime}\right) a\left(t^{\prime}\right)^{3} S\left(t^{\prime}\right)=0
$$

where

$$
\begin{aligned}
& R(t)=\dot{f}-2 H f+\frac{\mathbf{k}^{2}}{3 a^{2} H} f+a^{2} H f_{00}-\frac{8 \pi G_{N}}{3}\left(1+\frac{\rho}{\lambda}\right) \frac{a^{2}}{H} \tau_{00}, \\
& S(t)=\tau_{00}+3 H \tau_{(L) 0}+\frac{2 \mathbf{k}^{2}}{a^{2}} \tau_{(L L)} .
\end{aligned}
$$

Here, we have used the definition of four-dimensional effective Newton's constant $G_{N}$ and the four-dimensional effective cosmological constant $\Lambda_{4}$ introduced in section II.

\section{B. Standard cosmology}

Now, for the purpose of the comparison, let us review the gauge-invariant formalism of cosmological perturbations in the four-dimensional Einstein gravity [28 30] in terms of the 4-gauge-invariant variables used in the present paper. We consider scalar perturbations around the Friedmann-Robertson-Walker spacetime. Namely, we consider the metric

$$
d s_{4}^{2}=\left(q_{\mu \nu}^{(0)}+\delta q_{\mu \nu}\right) d y^{\mu} d y^{\nu}
$$

where

$$
q_{\mu \nu}^{(0)} d y^{\mu} d y^{\nu}=-d t^{2}+a^{2}(t) \sum_{i=1}^{3}\left(d y^{i}\right)^{2}
$$

and

$$
\delta q_{\mu \nu} d y^{\mu} d y^{\nu}=\int d^{3} \mathbf{k}\left[\sigma_{00} Y d t^{2}+2 \sigma_{(L) 0} V_{(L) i} d t d y^{i}+\left(\sigma_{(L L)} T_{(L L) i j}+\sigma_{(Y)} T_{(Y) i j}\right) d y^{i} d y^{j}\right]
$$

Here, the coefficients $\sigma_{00}, \sigma_{(L) 0}, \sigma_{(L L)}, \sigma_{(Y)}$ depend only on the proper time $t$. Following ref. [9], we can construct gauge-invariant variables $f_{00}$ and $f$. 


$$
\begin{aligned}
f_{00} & =\sigma_{00}-2 \dot{\chi}, \\
f & =\sigma_{(Y)}+2 a^{2} H \chi+\frac{2 \mathbf{k}^{2}}{3} \sigma_{(L L)},
\end{aligned}
$$

where $\chi=\sigma_{(L) 0}-\dot{\sigma}_{(L L)}+2 H \sigma_{(L L)}$.

As for the stress energy tensor, we consider

$$
T_{(4) \nu}^{\mu}=-\frac{\Lambda_{4}}{8 \pi G_{N}} \delta_{\nu}^{\mu}+T_{\nu}^{\mu}+\delta \tilde{T}_{\nu}^{\mu},
$$

where $\Lambda_{4}$ is the four-dimensional cosmological constant, $T_{\mu \nu}$ is given by (13) and

$$
\delta \tilde{T}_{\mu \nu} d y^{\mu} d y^{\nu}=\int d^{3} \mathbf{k}\left[\tilde{t}_{00} Y d t^{2}+2 \tilde{t}_{(L) 0} V_{(L) i} d t d y^{i}+\left(\tilde{t}_{(L L)} T_{(L L) i j}+\tilde{t}_{(Y)} T_{(Y) i j}\right) d y^{i} d y^{j}\right] .
$$

Again, the coefficients $\tilde{t}_{00}, \tilde{t}_{(L) 0}, \tilde{t}_{(L L)}, \tilde{t}_{(Y)}$ depend only on the proper time $t$. Following ref. [8], we can construct gauge-invariant variables $\tau_{00}, \tau_{L(0)}, \tau_{(L L)}$ and $\tau_{(Y)}$.

$$
\begin{aligned}
\tau_{00} & =\tilde{t}_{00}+\dot{\rho} \chi, \\
\tau_{(L) 0} & =\tilde{t}_{(L) 0}+\frac{1}{2}(\rho+p)\left[\chi-\dot{\sigma}_{(L L)}+2 H \sigma_{(L L)}\right], \\
\tau_{(L L)} & =\tilde{t}_{(L L)} \\
\tau_{(Y)} & =\tilde{t}_{(Y)}+a^{2} \dot{p} \chi .
\end{aligned}
$$

We can expand the four-dimensional Einstein's equation $G_{(4) \nu}^{\mu}=8 \pi G_{N} T_{(4) \nu}^{\mu}$ up to the first order in the perturbations, where $G_{(4) \nu}^{\mu}$ is the Einstein tensor constructed from the metric $q_{\mu \nu}$, and express it in terms of the above gauge-invariant variables. The background equations are, of course, the Friedmann equation

$$
H^{2}=\frac{8 \pi G_{N}}{3} \rho+\frac{\Lambda_{4}}{3}
$$

and the conservation equation

$$
\dot{\rho}+3 H(\rho+p)=0 .
$$

The first order equations are

$$
\begin{aligned}
\dot{f}-2 H f+\frac{\mathbf{k}^{2}}{3 a^{2} H} f+a^{2} H f_{00} & =\frac{8 \pi G_{N}}{3 H} a^{2} \tau_{00}, \\
\dot{f}-2 H f+a^{2} H f_{00} & =-8 \pi G_{N} a^{2} \tau_{(L) 0}, \\
a^{2} f_{00}-f & =32 \pi G_{N} a^{2} \tau_{(L L)}, \\
\ddot{f}-H \dot{f}-2\left(H^{2}+\dot{H}\right) f+\frac{\mathbf{k}^{2}}{3 a^{2}} f+a^{2} H \dot{f}_{00}+a^{2}\left(3 H^{2}+2 \dot{H}\right) f_{00}-\frac{\mathbf{k}^{2}}{3} f_{00} & =-8 \pi G_{N} \tau_{(Y)} .
\end{aligned}
$$

These four equations are equivalent to the following set of four equations.

$$
\begin{gathered}
\dot{\tau}_{(L) 0}+3 H \tau_{(L) 0}-\frac{1}{a^{2}} \tau_{(Y)}+\frac{4}{3} \frac{\mathbf{k}^{2}}{a^{2}} \tau_{(L L)}+\frac{1}{2}(\rho+p) f_{00}=0, \\
\dot{\tau}_{00}+3 H \tau_{00}+\frac{3 H}{a^{2}} \tau_{(Y)}+\frac{\mathbf{k}^{2}}{a^{2}} \tau_{(L) 0}+\frac{3}{2 a^{2}}(\rho+p)(\dot{f}-2 H f)=0, \\
\ddot{f}+a^{2} H \dot{f}_{00}+2\left(2 H^{2}+\dot{H}\right)\left(a^{2} f_{00}-f\right)+\frac{\mathbf{k}^{2}}{3 a^{2}}\left(2 f-a^{2} f_{00}\right) \\
+8 \pi G_{N} \tau_{(Y)}-\frac{8 \pi G_{N}}{3} a^{2} \tau_{00}=0,
\end{gathered}
$$

and 


$$
\dot{f}-2 H f+\frac{\mathbf{k}^{2}}{3 a^{2} H} f+a^{2} H f_{00}-\frac{8 \pi G_{N}}{3} \frac{a^{2}}{H} \tau_{00}=0 .
$$

Finally, in the standard cosmology, there are two well-known algebraic relations among gauge-invariant variables derived from the perturbed Einstein's equation. With our notation, those are

$$
\begin{aligned}
\mathbf{k}^{2} f & =8 \pi G_{N} a^{4}\left(\tau_{00}+3 H \tau_{(L) 0}\right), \\
a^{2} f_{00}-f & =32 \pi G_{N} a^{2} \tau_{(L L)} .
\end{aligned}
$$

\section{Similarity and difference}

The equations (65) in the standard cosmology are nothing but the perturbed conservation equation and, of course, exactly the same as those for the brane-world cosmological perturbations (50). The equation (66) in the standard cosmology is similar to the equation (51) in the brane-world cosmology. In particular, in the low energy limit when $|\rho| / \lambda \ll 1$ and $|p| / \lambda \ll 1$, these equations become identical. The final equation (67) in the standard cosmology is, in some sense, similar to the integro-differential equation (52). Actually, in a situation when the effect of the bulk gravitational waves (the integral in the integro-differential equation) is negligible $\$$, the integro-differential equation is reduced to $R(t) \simeq 0$, which becomes the same as (67) in the low energy limit.

However, in general, non-local properties of the integro-differential equation (52) in the brane-world cosmology are expected to become significant. Moreover, in high energy, corrections of order $O(\rho / \lambda)$ and $O(p / \lambda)$ become significant, too. In particular, in the brane-world cosmology we have not found simple algebraic relations like (58). Hence, there is a good possibility that we can find observable differences between the brane-world cosmology and the standard cosmology, by analyzing the set of equations (52), (51) and (50). Furthermore, if we consider the initial gravitational waves in the bulk $\left(\Phi_{\text {in }} \neq 0\right)$ then these waves affect the evolution of perturbations on the brane as expressed by the more general integro-differential equation (48).

\section{SUMMARY}

We have analyzed cosmological perturbations in the Randall-Sundrum brane-world scenario with a positive tension brane in the AdS background bulk geometry by using the doubly gauge-invariant formalism. For simplicity, we have considered scalar perturbations around a plane symmetric $(K=0)$ background. We have derived the set of four coupled equations (42), (43) and (48). The final equation is an integro-differential equation whose kernel is constructed formally from the $\mathbf{Z}_{2}$-symmetric retarded Green's function of the bulk gravitational waves. Hence, this equation describes non-local properties due to the gravitational waves propagating in the bulk. Finally, we compare these equations with the corresponding equations in the standard cosmology. In particular, we have seen that the number of independent equations, four, is the same as in the standard cosmology and that in low energy these sets of equations differ only by the non-local effects due to gravitational waves in the bulk.

Although we have succeeded in deriving the integro-differential equation formally, we need an explicit expression of the $\mathbf{Z}_{2}$-symmetric retarded Green's function in order to obtain the kernel. Since evaluation of the Green's function for a general motion of the brane seems difficult, it might be more realistic to adopt numerical methods to integrate the master equation (32) in the bulk and the three differential equations on the brane, (42) and (43), at the same time. In this case, we can use the equations (45) and (44) to give a boundary condition to $\Phi$ and to give a feedback of $\Phi$ to perturbations on the brane. Namely, we have one equation for bulk perturbations, three equations for perturbations on the brane and two equations to relate these two kinds of perturbations.

The author would like to thank Werner Israel for continuing encouragement and helpful discussions. This work was supported by the CITA National Fellowship and the NSERC operating research grant.

\footnotetext{
${ }^{8}$ Actually, in the static background $H=0$, the so called zero-mode truncation [32] corresponds to setting $\bar{G}_{\mathbf{R}}\left(t, t^{\prime}\right) \simeq 0$
} 
[1] L. Randall and R. Sundrum, Phys. Rev. Lett. 83, 4690 (1999) hep-th/9906064.

[2] J. M. Cline, C. Grojean and G. Servant, Phys. Rev. Lett. 834245 (1999) hep-ph/9906523.

[3] E. E. Flanagan, S. H. H. Tye, I. Wasserman, Phys. Rev. D62, 044039 (2000) thep-ph/9910498.

[4] P. Binétruy, C. Deffayet, U. Ellwanger and D. Langlois, Phys. Lett. B477, 285 (2000) hep-th/9910219].

[5] S. Mukohyama, Phys. Lett. B473, 241 (2000) hep-th/9911165.

[6] P. Kraus, JHEP 9912, 011 (1999) hep-th/9910149.

[7] D. Ida, JHEP 0009, 014 (2000) gr-qc/9912002.

[8] S. Mukohyama, Phys. Rev. D62, 084015 (2000) hep-th/0004067.

[9] S. Mukohyama, Class. Quant. Grav. 17, 4777 (2000) hep-th/0006146].

[10] H. Kodama, A. Ishibashi and O. Seto, Phys. Rev. D62, 064022 (2000) hep-th/0004160].

[11] H. Kodama, hep-th/0012132.

[12] R. Maartens, Phys. Rev. D62, 084023 (2000) hep-th/0004166; C. Gordon and R. Maartens, Phys. Rev. D63, 044022 (2001) hep-th/0009010; R. Maartens, V. Sahni and T. D. Saini, Phys. Rev. D63, 063509 (2001) gr-qc/0011105.

[13] D. Langlois, Phys. Rev. D62, 126012 (2000) [ hep-th/0005025]; Phys. Rev. Lett. 86, 2212 (2001) [hep-th/0010063].

[14] C. van de Bruck, M. Dorca, R. Brandenberger and A. Lukas, Phys. Rev. D62, 123515 (2000) [hep-th/0005032]; C. van de Bruck and M. Dorca, hep-th/0012073; M. Dorca and C. van de Bruck, hep-th/0012116.

[15] K. Koyama and J. Soda, Phys. Rev. D62, 123502 (2000) [hep]

[16] D. Langlois, R. Maartens and D. Wands, Phys. Lett. B489, 259 (2000) hep-th/0006007.

[17] D. Langlois, R. Maartens, M. Sasaki and David Wands, Phys. Rev. D63, 084009 (2001) hep-th/0012044.

[18] S. Mukohyama, T. Shiromizu and K. Maeda, Phys. Rev. D62, 024028 (2000), Erratum-ibid. D63, 029901 (2001) [hepth/9912287.

[19] D. Birmingham, Class. Quant. Grav. 16, 1197(1999) hep-th/9808032.

[20] S. W. Hawking, Commun. Math. Phys. 43, 199 (1975).

[21] W. D. Goldberger and M. B. Wise, Phys. Rev. Lett. 83, 4922 (1999) hep-ph/9907447.

[22] O. DeWolfe, D. Z. Freedman, S. S. Gubser and A. Karch, Phys. Rev. D62, 046008 (2000) [hep-th/9909134].

[23] S. Mukohyama, Phys. Rev. D63, 044008 (2001) hep-th/0007239].

[24] S. Mukohyama, Phys. Rev. D, to appear [hep-th/010103\&].

[25] W. Israel, Nuovo Cim. B44, 1 (1966); Erratum-ibid. B48, 463 (1967).

[26] T. Shiromizu, K. Maeda and M. Sasaki, Phys. Rev. 62, 024012 (2000) gr-qc/9910076.

[27] T. Tanaka and X. Montes, Nucl. Phys. B582, 259 (2000) hep-th/0001092.

[28] J. M. Bardeen, Phys. Rev. D22, 1882 (1980).

[29] H. Kodama and M. Sasaki, Prog. Theor. Phys. Suppl. 78, 1 (1984).

[30] V. F. Mukhanov, H. A. Feldman, and R. H. Brandenberger, Phys. Rep. 215, 203 (1992).

[31] GRTensor II, http://grtensor.phy.queensu.ca.

[32] J. Garriga and T. Tanaka, Phys. Rev. Lett. 84, 2778 (2000) hep-th/9911055. 